\title{
La vie végétative des animaux : la destruction heideggérienne de l'animalité
}

\author{
$\mathrm{C}_{\text {hristiane }} \mathrm{B}_{\text {alley }}$
}

«Ici peut sans doute surgir la vaste question : d'où savons-nous ce qui se passe dans l'animal et ce qui ne s'y passe pas? Nous ne pouvons le savoir immédiatement, mais nous pouvons néanmoins acquérir médiatement une certitude métaphysique sur l'être-animal ». (Heidegger, Qu'est-ce qu'une chose?)

«Le saut de l'animal qui vit à l'homme qui dit est aussi grand sinon encore plus grand que celui de la pierre sans vie à l'être vivant». (Heidegger, Les hymnes de Hölderlin)

«From both sides of the gully, Heidegger claims to catch sight of what so few mortals have caught sight of, namely, the fact that the signless animal is penned in so tightly that even its traditional self-animation must be denied it ». (Krell, Daimon Life)

Dans Être et temps, l'homme comme être-au-monde se distinguait des choses inanimées (des étants Vorhandene et Zuhandene), mais la question de l'être des animaux et de l'être de la vie en général, était laissée « ontologiquement indéterminée » $(\mathrm{SZ}, \S 10,50 \text { [59] })^{1}$. Deux ans plus tard, dans son cours sur les Concepts fondamentaux de la métaphysique. Monde, finitude, solitude, Heidegger n'expose plus l'homme comme être-au-monde, mais bien comme configurateur de monde, comme weltbilden ${ }^{2}$. S'essayant à «un nouveau départ », il tente une nouvelle approche du phénomène du monde par le moyen d'un examen comparatif de trois thèses : «la pierre est sans monde (weltlos) », «l'animal est pauvre en monde (weltarm) », «l'homme est configurateur de monde (weltbilden)»(GA 29/30, 277). De par sa configuration du monde, l'homme se distingue alors non plus de la nature inanimée, mais bien de tous les 
animaux et de tous les vivants, ontologiquement déterminés par la weltarmüt, la pauvreté en monde.

À première vue, il semble donc que l'essence de l'homme, afin de bien se démarquer non plus seulement des choses, mais bien cette fois des animaux, ait dû subir un certain déplacement : de l'In-der-Welt-Sein à la Weltbildung, de l'être-au-monde à la configuration en monde. Ce soupçon habite la réception—essentiellement critique—de l'ontologie heideggérienne de la vie animale : Heidegger se verrait contraint de revenir en deçà des avancées d'Être et temps en réhabilitant la primauté du logos apophantique au détriment du logos herméneutique afin de maintenir l'abîme zooanthropologique ${ }^{3}$. Nous devons bien avouer que la manière dont Heidegger explicite l'essence de l'homme en termes de «tenue en vis-à-vis du monde » (266) et de tenuede-rapport avec «l'étant en tant qu'étant et dans son entièreté » (412) détonne considérablement avec l'être-au-monde englué et accaparé dont les analyses phénoménologiques ont fait la fortune d'Être et temps. D'autre part, la publication des cours de Heidegger antérieurs à Être et Temps ne fait que renforcer cette hypothèse puisqu'il y reconnaît à plusieurs reprises l'animal comme un être-au-monde : «Tout vivant, dans la mesure où il est, a un monde, ce qui ne vaut pas pour le non-vivant ${ }^{4}$. $\mathrm{Si}$ «l'essentiel est manqué si je ne vois pas que l'animal a un monde », si «toute vie est là de telle sorte qu'un monde est également là pour elle ${ }^{5} »$, il semble que l'essence de l'homme doive alors être déterminée d'une manière plus spécifique.

Et pourtant, c'est une tout autre histoire qui se raconte dans le cours de 1929-1930 : ce n'est pas l'essence de l'homme qui subit un déplacement, mais bien plutôt l'essence de l'animal. Passant de l'être-au-monde qu'il était jusqu'en 1926 à cet être privé de monde qu'il sera à partir des années 1930, l'animal se voit privé de tout ce qui l'a toujours caractérisé en propre: la faculté de percevoir, de se sentir, de se mouvoir et de désirer. Parler ici d'une déconstruction de 
l'essence de l'animalité serait un euphémisme puisque c'est véritablement une destruction pure et simple de l'idée d'animalité qui a lieu dans l'ontologie heideggérienne de la vie. Comme nous le verrons ici, Heidegger se livre à une réduction méthodique et systématique de tout ce qui chez les animaux a, de près ou de loin, à voir avec nous-mêmes, à tel point qu'au terme du cours de Fribourg, il n'y a plus de différence ontologiquement décisive entre une vie végétale et une vie animale, entre une vie végétative et une vie de relation caractérisée par la sensibilité et la mobilité.

\section{La pauvreté animale comme privation en monde (Weltarmut als Weltentbehren)}

À la lueur des indications d'Être et temps, la caractérisation des animaux par la pauvreté en monde ira de soi : puisque nous ne pouvons avoir accès à l'essence de la vie qu'au fil d'une «interprétation privative » (SZ 50 [59]) où il s'agit, comme le précisera Heidegger, «de défaire couche par couche ce qui est en excès par rapport à elle » (GA 29/30, 372), nul étonnement que les animaux apparaissent sous figure de manque, de pauvreté. En revanche, il sera très étonnant d'apprendre que cette thèse s'oriente sur « un regard porté sur l'animalité elle-même » (291) et qu'elle «n'établit pas d'ordre hiérarchique» (287). Heidegger y insiste longuement : «cette comparaison entre l'animal et l'homme dans l'optique de la pauvreté en monde et de la configuration en monde ne permet aucune estimation ni aucune évaluation de perfection et d'imperfection » (289). La pauvreté en monde de l'animal ne signifie pas que l'animal a moins de monde, mais signifie au contraire que l'animal n'a pas de monde, même pas un peu : «Être pauvre signifie : être privé» (290).

Si la pauvreté en monde doit être conçue comme une privation en monde, si la Weltarmüt est en réalité Weltentbehren, pourquoi n'avoir pas alors dit que l'animal était, comme la pierre, 
tout simplement sans monde (Weltlos)? Comme nous le savons, Heidegger n'hésitera pas à le faire par la suite ${ }^{6}$, mais dans le cours de 1929-1930, il refuse d'entendre la privation en monde de l'animal comme un simple non avoir, puisque s'il n'a pas de monde, «l'animal n'est pas non plus quelque chose qui se trouverait simplement être là : il est dans son mode d'être, déterminé par le fait qu'il a accessibilité à... »(297). À la différence d'une pierre gisant là sur le sol, chauffée par le soleil, dont l'être est essentielle absence d'accès à quoi que ce soit, un lézard étendu sur cette même pierre a un rapport complètement différent à la pierre, au sol et au soleil. Le lézard a bien, contrairement à la pierre, accès à quelque chose, il a une relation propre aux choses aux alentours, au soleil et à la roche. Toutefois, et là sera le point principal de Heidegger, ni le soleil, ni la roche ne lui sont donnés comme soleil et comme roche :

Quand nous disons que le lézard est allongé sur la roche, nous devrions raturer le mot « roche » pour indiquer que ce sur quoi le lézard est allongé lui est certes donné d'une façon ou d'une autre mais n'est pas reconnu comme roche. La rature du mot ne signifie pas seulement : prendre quelque chose d'autre comme quelque chose d'autre. La rature signifie plutôt que la «roche» n'est absolument pas accessible comme étant. (GA 29/30, 294)

Nous avons ici l'essentiel de l'argumentaire de Heidegger sur l'essence de l'animalité : l'homme, du haut de sa configuration en monde, n'a pas davantage de monde et l'animal, du fond de sa pauvreté, moins. Le terme de «pauvreté » cherche à exprimer le fait qu'il y a dans l'animal un avoir et un non avoir : «Certes, l'animal a un accès à... et un accès à quelque chose qui est réellement — mais ce quelque chose, c'est seulement nous qui sommes capables de l'éprouver et de le voir se manifester en tant qu'étant » (390). 


\section{Interprétation traditionnelle de la privation en monde de l’animal}

Qu'entend exactement Heidegger en disant que l'animal n'a pas accès à l'étant « comme tel » (als solchem) ou « comme étant» (als Seiendem)? Assurément cela signifie que l'animal est situé hors de l'appréhension de l'être de l'étant. Mais qu'est-ce que cela implique concrètement? L'animal a-t-il tout de même la possibilité d'entrer en relation avec l'étant comme étant, par exemple, menaçant ou attirant? Tout cela semble tellement évident que l'affaire est déjà classée : les animaux font l'expérience de l'étant d'une certaine manière, ils perçoivent différemment les mêmes choses que nous, ils discriminent ou considèrent les choses selon leur propre « échelle », «leur propre mesure »: les choses leur apparaissent toujours comme ceci ou cela et pour ceci ou cela, dans l'orée de leurs préoccupations immédiates, mais ils sont par principe privés de la possibilité de se déprendre de cette significativité immédiatement impérative qui leur impose un rapport déterminé avec l'étant. Comme le posera V. Houillon : «L'animal se rapporte à de l'étant dans la recherche de l'étant comme nourriture, comme proie ou ennemi, comme matériau de construction d'un nid ou d'un abri. L'animal se rapporte à son monde d'animal selon une inaccessibilité au monde comme phénomène du monde » (Houillon 123) ${ }^{7}$. La pauvreté en monde signifierait qu'un animal vit dans « un contexte minimal de signifiance » (White $2 \mathrm{n})^{8}$ dans lequel il est trop entièrement englué pour pouvoir «détacher les choses de leur utilité immédiate » (Burgat 120). «Incapable de recul par rapport aux objets qui l'entourent », l'animal ne peut avoir « un rapport autre qu'utilitaire aux choses » (Burgat 139 et 128). Malgré les difficultés évidentes

qu'impliquent une telle interprétation de la pauvreté en monde comme une forme d'engloutissement affairé dans un milieu ambiant dont l'animal ne peut, par principe, se déprendre, alors que l'homme serait capable de mettre ce monde à distance pour le considérer 
comme tel, comme monde, il n'en demeure pas moins que c'est pourtant bien ainsi qu'a été, le plus souvent, comprise la thèse heideggérienne. Ainsi, William McNeill, au terme de ce qui est sans doute l'analyse la plus élaborée du cours de 1929-1930 avec celle D. F. Krell, suppose que ce qui est enquêté ici est la possibilité de la connaissance métaphysique et scientifique, et il en vient à la conclusion que les animaux sont incapables de prendre une "position libre et indépendante » vis-à-vis de l'étant (McNeill 239 et 246). C'est également dans une telle voie interprétative que s'engouffrera Derrida - non sans pressentir la mer de difficultés que cette thèse implique - en ramenant continuellement la privation de l'animal à la question de l'objectivité 9 .

Pourtant, si c'est bien de la possibilité générale d'appréhender l'étant hors de tout projet dont Heidegger prive les bêtes, la thèse de la pauvreté en monde de l'animal conçue comme privation en monde est difficilement conceptualisable : l'animal ne serait pas pauvre en monde au sens où Heidegger l'entend, c'est-à-dire au sens de privé, mais pauvre au sens usuel du terme. Ainsi, l'animal habiterait simplement plus pauvrement que l'homme, car privé de cette «tenue en vis-à-vis », de cette retenue, de ce « recul », disait Scheler, par lequel un Umwelt devient Welt, incapable d'accomplir cette neutralisation du monde ambiant et de se déprendre du réseau de significativités et de rapports dans lequel il est englué, afin de s'appréhender comme tel, comme être-englué. Cette existence appauvrie de l'animal ferait néanmoins de lui un être «à la mesure du Dasein»(Daseinsmässig). Puisque nous sommes nous-mêmes d'ordinaire «entièrement auprès de ce qui nous préoccupe » de telle sorte que «[n]ous sommes pris (hingenommen) par les choses, si ce n'est perdus en elles, souvent même étourdis (benommen) par elles » (GA 29/30, 158), ne faut-il pas en conclure que l'abîme ontologique qui sépare les hommes et animaux est ontiquement toujours-déjà franchi? 
Nul étonnement, à la lueur de ces interprétations, que l'ontologie heideggérienne de la vie ait été généralement considérée comme un échec, voire comme le «plus splendide échec de Heidegger » (Krell 8). En définissant l'essence de l'animal par la pauvreté en monde, Heidegger aurait doublement échoué. Non seulement la manière dont il définit l'essence de l'animalité serait traditionnelle (penser l'animal en ce qu'il lui manque pour être homme), mais le contenu de la privation serait tout aussi traditionnel : l'animal serait, encore une fois, privé du logos, au sens étroit de « raison», de rapport objectif à l'étant. Ce faisant, Heidegger aurait ainsi échoué à deux tâches qu'il se donnait explicitement dans le cours de Fribourg : celle d'effectuer un « renversement complet de notre conception de l'homme»(101), ainsi que celle de «s'assurer primordialement de la vie à partir d'elle-même et dans sa teneur essentielle » et non plus, comme nous l'avons toujours fait, à partir de l'homme ou de la matière inanimée (286). Au bout du compte, dans la figure essentiellement privative de l'animal qui se dégagerait de son ontologie de la vie, Heidegger trahirait son anthropocentrisme, son logocentrisme, son dogmatisme et son humanisme récessif, ses «affinités troublantes » avec Hegel, et, plus généralement, son échec à déconstruire la tradition métaphysique ${ }^{10}$. C'est alors que la prétention heideggérienne à avoir accompli une «mise hors circuit de la définition traditionnelle et confirmée de l'homme » (SZ 183 [142]) apparaîtrait alors bien vaine et fort présomptueuse, déconstruction qu'il réitère pourtant dans son cours de 1929-1930 comme une «exigence » (101).

\section{L'animal vit hors différence ontologique}

La plus grande difficulté à laquelle nous sommes confrontés tient en ce que la distinction phare d'Etre et temps entre existence inauthentique et existence authentique disparaît comme telle dans le cours sur Les concepts fondamentaux de la métaphysique, alors que c'est bien là que 
se situe le nerf du problème: qu'est-ce qui différence la manière dont l'animal se trouve accaparé et obnubilé (benommen) par son milieu ambiant, de la manière dont l'homme est luimême quotidiennement «capté (benommen) par le monde dont il se préoccupe » (SZ 61 [66])? Ne sommes-nous pas nous-mêmes le plus souvent pauvres en monde, comme le soupçonne Derrida? La question principale consiste à déterminer si, pour Heidegger, l'animal se comporte dans son milieu ambiant en percevant toujours comme tel ou tel, pour ceci ou cela ce avec quoi il est en relation, mais incapable de l'appréhender comme étant simplement là. Dans le cours de 1929-1930, Heidegger peut sembler esquiver la question : «Il ne s'agit pas de savoir si l'animal prend autrement le donné ni comment il le fait, mais de savoir s'il peut ou non percevoir [Vernehmen] quelque chose en tant que tel, percevoir quelque chose en tant qu'étant» (GA 29/30, 383). Or, nous montrerons ici que Heidegger, répondant à la seconde question, répondait par là même à la première, puisque «c'est là seulement où, d'une façon générale, de l'étant se manifeste en tant qu'étant qu'il est possible d'éprouver tel ou tel étant déterminé en tant qu'il est ceci ou cela» (397; nous soulignons). Il faut prendre la mesure de l'importance primordiale de l'appréhension de l'être de l'étant : l'animal, privé de la possibilité de percevoir comme étant ce avec quoi il est en relation, est par là même exclu de la possibilité de percevoir quelque chose comme ceci ou cela. Situé hors différence ontologique, l'animal est d'emblée exclu du monde du sens.

Pourtant, comme en témoigne la réception embarrassée du cours de Fribourg, nous avons bien du mal à saisir comment Heidegger peut en venir à priver l'animal de la possibilité de percevoir comme tel ou tel ce avec quoi il est en relation. De toute évidence, l'animal doit bien percevoir comme attirante la chose qu'il poursuit et comme menaçante ce qu'il fuit! Voilà 
précisément le point litigieux soulevé par William McNeill, l'un des traducteurs du cours de Fribourg :

Although Heidegger, so far as I am aware, nowhere clarifies this sufficiently, the animal in fact has and must have a certain ability to relate to something as something, although not, indeed, as being something. It is indeed a precondition of the animal's being able to move $[\ldots]$. A cat responds to the presence of a mouse as its potential dinner, or as something to play with; it responds to the presence of a dog as a potential threat-and not as its food. (McNeill 240)

McNeill met le doigt sur le cour du problème : il semble en effet que l'animal, s'il peut se mouvoir, doit pouvoir percevoir comme ceci ou cela ce avec quoi il est en relation, même s'il ne peut à l'évidence pas le percevoir comme étant ceci ou cela. McNeill en appelle ici à l'argumentaire aristotélicien selon lequel la perception (aisthesis) comme pouvoir de discrimination (krinein) est condition de possibilité du mouvement local (kinesis) propre à l'animal. En tant qu'il se meut — qu'il peut fuir ou poursuivre —, l'animal doit avoir part à une forme de discrimination élémentaire qui lui permet de percevoir l'étant comme indésirable ou désirable, comme menace ou proie, comme à-fuir ou à-poursuivre. Selon McNeill, Heidegger ne résout jamais vraiment le problème. Et pourtant, nous montrerons que là est précisément le sens de toutes ses analyses! Heidegger affirme sans équivoque : «C'est là seulement où, d'une façon générale, de l'étant se manifeste en tant qu'étant qu'il est possible d'éprouver tel ou tel étant déterminé en tant qu'il est ceci ou cela — éprouver au sens large, qui dépasse le simple fait de connaître : c'est éprouver au sens de faire l'expérience de l'étant»(GA 29/30, 397). On ne pourrait plus clairement dire que la circonspection quotidienne qui ouvre l'à-portée-de-la-main n'est possible que sur fond de précompréhension de l'être de l'étant! Le commerce préoccupé qui ouvre l'étant comme à-portée-de-la-main (Zuhandenheit), dont il est question dans l'analyse de la mondanéité de Sein und Zeit, est rendu possible par l'appréhension athématique de l'être de 
l'étant. Cet enracinement du sens dans l'éclaircie de l'être est l'un des axiomes les plus durables de la pensée de Heidegger, qu'il réitérera jusque dans les Séminaires des années 1960 : «s'occuper de l'étant n'est possible et compréhensible que par le déval à partir de l'être ${ }^{11} »$. Le réseau de renvoi ouvert par l'habitation, d'emblée significative et impliquée dans l'étant, trouve sa condition de possibilité — sa fondation — dans la (pré)compréhension de l'être de l'étant : «Tel est le sens des analyses célèbres et pourtant méconnues de l'ustensilité dans Être et temps » (Séminaire du Thor 150). La confusion manifeste dans la réception de l'ontologie heideggérienne de la vie entre l'appréhension de l'être de l'étant et l'objectivation de l'étant témoigne d'une profonde mécompréhension du but véritable de Heidegger dans l'ontologie fondamentale de 1927 : montrer qu'il y a dans nos comportements les plus quotidiens une précompréhension de l'être de l'étant. Cette appréhension athématique de l'être qui est impliquée dans tous nos comportements, voilà ce dont Heidegger prive les animaux dans le cours de 1929-1930. Il n’y a nul retour de l'apophantique, puisque c'est de l'Als-Struktur générale dont il s'agit tout le long: la perception de l'«étant en tant qu'étant » ne renvoie pas à l'étant « comme étant simplement là sans plus » (Nur-noch-Vorhanden), mais bien à la précompréhension de l'être de l'étant. Ce n'est que parce que nous méconnaissons le rôle fondamental - et fondateur - joué par la compréhension de l'être dans la pensée de Heidegger que nous avons pu interpréter la privation en monde de l'animal comme un retour de l'apophantique et de la primauté de la Vorhandenheit. Niant que l'animal soit initié à la structure élémentaire du «comme», privant l'animal de la possibilité de percevoir «comme étant »ce avec quoi il est en relation, Heidegger implique par là qu'il vit hors phénoménalité et hors sens. «[A]u monde est associé la manifesteté de l'étant en tant que tel, de l'étant en tant qu'étant. Cela implique qu'avec le monde va de pair cet énigmatique "en tant que", celui de l'en tant que tel (formellement parlant: "quelque chose en 
tant que quelque chose") — cet "en tant que" qui est radicalement fermé à l'animal » (GA 29/30, 397). L'appréhension de «quelque chose comme quelque chose », c'est bel et bien la structure de la compréhension herméneutique (SZ, § 32, 149)! Ce n'est nullement de la possibilité de connaître, mais bien de celle de comprendre dont se trouvent privées les bêtes : le lézard ne perçoit pas la roche comme roche, mais il ne l'appréhende pas non plus comme appui, comme chaude ou froide, humide ou sèche : «Si vous percevez quelque chose comme étant ceci ou cela - par exemple, la chose comme verre - cela doit vous être manifeste comme quelque chose qui est »(Zollikon Seminar 90). La différence ontologique est « la plus ancienne différenciation, plus ancienne qu'aucune autre, car lorsque nous différencions un étant d'un autre, cette plus ancienne différenciation a déjà eu lieu » (GA 33, 25 [33]). Ce n'est que si nous prenons la mesure du rôle déterminant de cette appréhension de l'être que l'argumentaire de Heidegger sur l'animal prend sens : parce qu'il ne perçoit pas l'étant comme tel, l'animal «ne se trouve pas du côté de l'être humain et n'a aucun monde »(GA 29/30, 391).

Il ne s'agit pas simplement d'une altérité qualitative du monde animal par comparaison au monde humain. Et, à plus forte raison, il ne s'agit pas de différences quantitatives en termes d'ampleur ou d'étendue. Il ne s'agit pas de savoir si l'animal prend autrement le donné ni comment il le fait, mais de savoir s'il peut ou non percevoir [Vernehmen] quelque chose en tant que tel, percevoir quelque chose en tant qu'étant. S'il ne le peut, c'est qu'il est alors séparé de l'homme par un abîme. (GA 29/30, 383)

La mobilité circonspecte qui ouvre un monde ambiant, un réseau de sens et de renvois, implique que l'être en question soit capable de discrimination et de différenciation (krinein). Or, cela n'est pas le lot des êtres «simplement vivants », car cette «capacité critique de distinguer ${ }^{12} »$, de discerner, de discriminer (en général, de faire des différences) n’est possible que grâce à la différence ontologique, «précisément la différence qui, finalement et fondamentalement, rend possible toute différenciation et tout être-différencié » (511). Ce qui équivaut finalement à dire, 
comme le reconnaît Heidegger, que l'animal ne perçoit par vraiment : «Au fond, l'animal n'a pas de perception (Im grunde das Tier hat keine Wahrnehmung). » (376, traduction modifiée). Là est précisément, nous le verrons, le sens véritable des analyses de la Benommenheit animale : il n'y a, dans le comportement de l'animal, aucune forme de discrimination; il y a réaction à des percepts, mais il n'y a pas de perception.

\section{La privation de la perception véritable : l'animal vit hors phénoménalité}

Nous touchons à un axiome, sinon à l'axiome fondamental, qui commande de part en part la conception heideggérienne de l'animalité: les animaux ne perçoivent pas réellement. Réaffirmant le mot d'ordre de toute la tradition phénoménologique — «toute perception est perception de»-, Heidegger soutiendra, dans les dernières heures de son cours, que cela ne signifie pas seulement que toute perception est intentionnelle (au sens d'être dirigée-vers), mais qu'elle est également rassemblante ou unifiante, elle est toujours «perception de quelque chose comme quelque chose». Abordant l'étant comme tel ou tel, la perception rassemble deux éléments en formant un tout, une entièreté : «A comme $\mathrm{B} » ;$ « $\mathrm{A}$ est $\mathrm{B}$ ». Dans la simple perception, il y a déjà une forme de rassemblement, d'unification ou de synthèse, c'est-à-dire qu'il y a déjà configuration de monde (450-452). Depuis ses toutes premières lectures d'Aristote, Heidegger a toujours soutenu que ce n'est pas le logos qui ouvre au monde, mais c'est l'aisthesis elle-même : le «concept fondamental de la sensibilité » signifie «en ouvrant, se laisser donner un monde, le laisser venir à la rencontre »(GA 22, 186 [205]). Le revirement par lequel l'animal passe de l'être-au-monde qu'il était jusqu'en 1926, à l'être privé de monde qu'il sera par la suite, n'est aucunement une forme de retour au $\log o s$ — et, qui plus est, à cette forme dérivée qu'est le logos apophantique - , mais consiste précisément en ce que les animaux ne sont désormais plus 
considérés comme des êtres qui perçoivent et se sentent, mais comme des êtres qui ne font que vivre : ils sont des êtres « seulement vivants » (Nur-noch-Lebenden) (SZ, § 68b, 346/242), «rien de plus que vie»(Nur-noch-leben) (SZ, § 9, 50/59). En lecteur assidu d'Aristote, Heidegger n'ignorait pas qu'il dépouillait alors les animaux de ce qui les a toujours caractérisés en propre, c'est-à-dire la perception et la sensibilité : «Ce qui différencie le ל̧́n $\mu$ óvov — le seulementvivre, le "végéter" — vis-à-vis du לoov — de l'être vivant au sens de l'animal —, c'est le fait

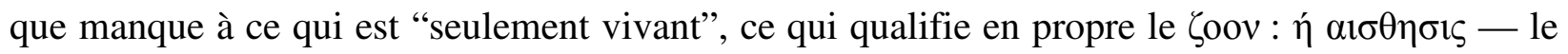
percevoir » (GA 33,124 [128]). Pourtant, Heidegger n'hésite pas affimer qu'il n’y a chez les animaux ni Vernehmen (GA 29/30, 361), ni Wahrnehmung (376). Comme il le fera à maintes reprises par la suite, il refuse toute comparaison entre la perception animale et humaine, arguant que «la vision, la faculté de voir propre à l'animal, est une aptitude, tandis que notre faculté de voir, en fin de compte, possède encore un tout autre caractère de possibilité et un tout autre genre d'être » $(338)^{13} . \mathrm{Si}$ «jamais la distinction entre l'animal et l'homme n'a été aussi radicale ni aussi rigoureuse dans la tradition philosophique occidentale que chez Heidegger » (Derrida, Points 289), c'est parce que ce n'est pas seulement le logos qui est refusé aux animaux, mais bel et bien «l'ouverture prélogique à l'étant » qui est déjà là dans la perception (GA 29/30, 490494). Dès lors qu'il est posé que «la perception chez l'animal est fondamentalement autre que celle de l'homme», on comprend qu' «il serait trompeur de penser qu'il suffit de retrancher la pensée et la raison pour avoir ce qu'a l'animal » (GA 33, 196 [194]).

Que nous ayons eu bien du mal, comme en témoigne la réception embarrassée de notre cours, à saisir le sens véritable des analyses de Heidegger est tout à fait compréhensible puisque cela a pour conséquence de priver les animaux de ce qui les a toujours définis en propre. Mais il n'en demeure pas moins qu'une telle mécompréhension de la radicalité de la position 
heideggérienne est étonnante puisque Richir, un des premiers à avoir offert une analyse exhaustive de ce cours au public français, avait déjà très bien saisi le sens de la privation en monde de l'animal : «la capture comportementale (Benommenheit) de l'animal est telle que la perception en général [...] y est impossible ». Heidegger ne prive pas l'animal d'un rapport à quelque chose d'objectif, mais bien d'un quelconque rapport à quelque chose de phénoménal : il n’y a pour l'animal «aucune phénoménalisation, aucun phénomène et aucune phénoménalité » (Richir 251-252).

Mais alors, comment les animaux peuvent-ils accomplir toutes leurs actions si complexes s'ils ne perçoivent pas véritablement et s'ils n'éprouvent rien, même «au sens large » du terme «éprouver» qui, précise Heidegger, «dépasse le simple fait de connaître » (397)? Pour le comprendre, nous devons d'abord saisir que pour Heidegger, le comportement animal ne nécessite pas la perception, mais au contraire, il s'y oppose: «il n'y a pas de perception (Vernehmen), mais un comportement (Benehmen)»(361). Ce n'est que si nous comprenons que Heidegger en vient à opposer le comportement à la perception que nous pouvons comprendre que l'animal fait tout ce qu'il fait sans que nous devions dire pour autant que l'animal perçoive véritablement. Ce sera la tâche de ce que nous appelons la «zoologie positive » de Heidegger.

\section{La « zoologie positive » de Heidegger : le double versant de la Benommenheit}

Cette zoologie positive, au terme de laquelle Heidegger prétend n'avoir fourni rien de moins que la «base sur laquelle peut s'établir toute question concrète en biologie » (377) est souvent passée sous silence, à tel point en fait que nous avons même pu affirmer que les analyses «patientes », «prudentes » et «embarrassées » de Heidegger aboutissent en définitive à un

«dead end»(Calarco 28). À trop accentuer le caractère aporétique du cours de Fribourg, on 
passe alors à côté du fait que Heidegger prétend bel et bien, au final, avoir élucidé l'essence de l'animalité d'une manière authentique : elle ne réside ni dans la pauvreté en monde (Weltarmüt), ni dans la privation en monde (Weltentbehren), puisque c'est en réalité la Benommenheit qui est considérée par Heidegger comme étant «la proposition de fond» de la métaphysique sur l'animal, et c'est elle qui finalement permet de maintenir — en la fondant — la thèse de la pauvreté en monde (394).

Nous insistons beaucoup trop dans la réception de ce cours sur l'échec de l'ontologie heideggérienne de la vie animale, car il faut voir que l'échec — et cela est crucial pour notre propos - concerne non pas les analyses de l'essence de l'animalité de l'animal, mais bien la thèse de la pauvreté en monde : troquer la caractérisation traditionnelle d'alogon pour de celle de weltarm ou de weltentbehren, c'est bien entendu encore et toujours penser l'animal en ce qui lui manque pour être homme. Or, Heidegger n'a pas manqué de s'en apercevoir lui-même : c'est précisément en raison de l'anthropocentrisme inhérent à une telle caractérisation qu'il médite si longuement la thèse de la pauvreté en monde de l'animal, soulevant à son propos «le doute le plus radical »(392), et qu'au final il y renonce - du moins, en tant que candidate à une définition authentique de l'essence de l'animalité. Si Heidegger insiste malgré tout pour maintenir la thèse de la pauvreté en monde de l'animal après avoir reconnu son caractère traditionnel, anthropocentrique et uniquement comparatif, c'est notamment parce que le concept de pauvreté explicite bien le double versant de l'essence de l'animalité, le fait qu'il y a, chez l'animal, un avoir et un non-avoir : «être ouvert dans l'hébétude (Benommenheit), c'est ne pas avoir de monde tout en ayant ce qui désinhibe » (389). Si nous restons à ce point obnubilés par le caractère traditionnel et aporétique des analyses de Heidegger, c'est que nous insistons beaucoup trop sur le versant négatif — «ne pas avoir de monde » - et trop peu sur le versant positif- 
«avoir ce qui désinhibe»-, ce qui est pourtant, dira Heidegger, « un avoir essentiel » du vivant. Le grand défi de Heidegger a été de ne pas s'en tenir à une caractérisation purement négative de l'animalité, de ne pas s'en tenir à une «zoologie privative » qui ne parle de l'animal que pour commander de n'en pas parler (Dastur 306), mais d'avoir tenté de montrer positivement en quoi consiste l'en vue de quoi et le comment de l'ouverture animale. Ou plutôt, de l'ouverture vitale, puisque Heidegger n'entend pas seulement élucider le mode d'être des animaux — de «tous les animaux, de chaque animal» (279) —, mais bien de tous les êtres vivants : l'objet de l'investigation est l'essence de la vie, «c'est-à-dire la manière d'être de l'animal et de la plante $\gg(281,286$, etc. $)$.

\section{Le comportement comme emprise pulsionnelle (die Benehmen als Benommenheit)}

Le problème est avant tout un problème de méthode: «De quelle manière la vie, l'animalité de l'animal et l'essence végétale des plantes doivent-elles nous devenir accessibles? » (270). D'entrée de jeu, Heidegger renonce à la possibilité «d'explorer les processus physiologiques et d'y associer en outre une quelconque psychologie animale », arguant que ce faisant, «nous avons déjà présupposé que l'animal vit, que d'une certaine manière il se sent, lors de ses agissements, du même coup de telle ou telle façon » (270). Pour Heidegger, il s'agit précisément de ne penser l'animal à partir de nous-mêmes (comme nous le faisons dans l'empathie naïve) sans pour autant penser l'animal à partir de la nature matérielle (comme le fait le mécanisme) : déplorant que nous pensions toujours la vie à partir d'autre chose, il affirme qu'il s'agit plutôt «d'élucider la vie à partir d'elle-même et dans sa teneur essentielle » (286). En conséquence, afin d'éviter de courir «le risque d'interpréter l'être de l'animal à partir de nousmêmes » (298), les analyses heideggériennes prennent leur point de départ dans la détermination 
de l'animal comme organisme: «tout ce qui est vivant est organisme» (313). Cette thèse directrice occupe Heidegger une bonne partie du cours, et ce n'est qu'après avoir longuement argumenté contre l'assimilation l'organisme avec la machine (§ 51 à § 57) qu'il se tourne vers l'élucidation de l'essence de la vie comme Benommenheit (§ 58 à $§ 60)$.

Formellement définie, la Benommenheit se décline selon un double mouvement: «Accaparement de l'animal veut donc dire à la fois : essentiel retrait de toute possibilité de percevoir quelque chose comme quelque chose, et, dans ce retrait précisément, être entraîné par...»(361). Le premier mouvement, sur lequel nous nous sommes longuement attardés, signifie qu' 'est prise à l'animal, dans son rapport à autre chose, la possibilité de se rapporter et de se relier à cette autre chose en tant que telle ou telle chose, en tant que chose se trouvant être là, en tant qu'étant» (361). Le sens, principalement négatif, du premier versant de la Benommenheit animale consiste donc à soutenir que l'animal est exclu de la phénoménalité, de la manifesté de l'étant (Offenbarkheit von Seiendem). L'étant n'est pas manifeste (offenbar) à l'animal, l'étant n'est pas ouvert (aufgeschlossen) pour lui, et pourtant, nous ne pouvons pas non plus affirmer que l'étant est pour lui simplement fermé (verschlossen) : «nous sommes bien obligés de dire que [...] le comportement atteste une ouverture à... (Offenheit für...)» (362). Mais ouverture à quoi, si ce n'est à l'étant? La réponse de Heidegger est en deux volets : l'animal est ouvert à des désinhibiteurs, des stimuli déclencheurs (§ 59), toujours sur le mode de la mise à l'écart (§ 60).

\section{L'en vue de quoi de l'ouverture animale : le cercle de désinhibition}

«Le comportement animal n'est pas une façon de faire et d'agir, comme l'est la tenue humaine d'un rapport (Verhalten), mais c'est un mouvement pulsionnel (Treiben).» (347). Il 
s'agit d'une «activité instinctive » (McNeill), d'une «capture comportementale » (Richir) où «est prise la possibilité de percevoir », mais où est donnée la possibilité d'être entraîné (hingenommen), d'être obnubilé (benommen), d'être pris par un cercle de désinhibition (Enthemmungsring). Afin de l'illustrer, Heidegger donne, au $§ 59 c$, l'exemple d'une abeille qui va butiner une fleur, s'arrête et retourne à la ruche, et il se demande: «Pourquoi l'abeille s'envole-t-elle et retourne-t-elle à la ruche? » Constate-t-elle qu'il n'y a plus de miel (Nichtmehrvorhandensein)? «Si c'est le cas, répond Heidegger, alors l'abeille doit avoir préalablement constaté que du miel se trouvait être là (als vorhandenen) » (352). S'il n'y aucune perception du miel comme étant là ou n'étant pas là, que se passe-t-il, pourquoi l'abeille cesse-telle de manger pour retourner à la ruche? La réponse de Heidegger est très simple, mais néanmoins énigmatique : la pulsion vers la nourriture a été commutée par une autre pulsion, c'est-à-dire qu'elle a été inhibée par la désinhibition d'une autre pulsion $(353)^{14}$. Heidegger parlera d'«inhibition par saturation» (Sättigungshemmung) (354): la saturation de la faim délivre l'insecte de la «pulsion fondamentale » (Grundtriebe) vers la nourriture et déclenche le mouvement de retour à la ruche. L'abeille rassasiée est donc poussée à retourner à la ruche. Mais comment retourne-t-elle à la ruche? De quelle manière s'y prend-t-elle? Ici, les choses se compliqueront considérablement : Heidegger soutient que l'abeille ne s'oriente pas, mais qu'elle est simplement prise par une «poussée directionnelle»(Richtungsgetriebenheit) qui a pris le relais de la pulsion précédente ${ }^{15}$. Arguant que «l'abeille ne prend pas une direction [...]; au contraire, elle est prise par une direction, la faisant jaillir en la poussant hors d'elle-même », Heidegger en vient à la conclusion qu'elle est simplement livrée (übergelassen) à ce que le soleil provoque chez elle (359-360). 
Complètement pris dans un jeu d'inhibitions (Hemmungen) et de désinhibititions (Enthemmungen), l'animal ne se rapporte pas à des choses particulières telles que la ruche ou l'endroit où se trouve la nourriture, mais il est constamment «tiraillé au sein d'une multiplicité de pulsions » et ainsi «suspendu entre lui-même et l'environnement (Umgebung)» (362). Cette « zone de compulsion des pulsions » (371) où une pulsion est inhibée par la désinhibition d'une autre délimite — d'une manière très précise, insiste Heidegger (374) — ce qui pourra venir jouer un rôle effectif, ce à quoi l'animal sera sensible, ou plutôt, pour être plus juste, ce à quoi il sera réceptif. Cette totalité de désinhibiteurs, de stimuli déclencheurs, forme un encerclement que Heidegger nommera Enthemmungsring et qui constitue l'Umgebung de l'animal, son environnement. Ce cercle de désinhibition (Enthemmungsring) est théorisé comme un anneau (Ring) - Heidegger parle d' « un tuyau qui ne s'élargit ni ne se resserre » (295) — dans lequel l'animal est enfermé pour toute la durée de sa vie et qui «trace par avance » ce que l'animal pourra rencontrer comme incitations ou, plutôt, comme excitations, c'est-à-dire ce qui sera susceptible de déclencher ou de désinhiber une pulsion.

\section{La pulsion animale (Trieb) n'est pas une impulsion (Drang)}

Mais une question ne manque pas de se poser ici : pourquoi la pulsion (Trieb) a-t-elle besoin d'être dés-inhibée? Ne l'est-elle pas d'emblée? Heidegger est bien conscient que c'est «précisément l'absence d'inhibition qui est le signe des pulsions », de telle sorte que nous disons que «ce qui inhibe la pulsion, c'est ce à quoi l'animal se heurte » (370). Et pourtant, il insiste tout de même pour dire que l'animal rencontre non pas des inhibiteurs (Hemmungen : des entraves, des résistances), mais bien des désinhibiteurs (Enthemmungen: des excitants, des 
stimuli déclencheurs). Heidegger conceptualise en effet la pulsion d'une manière très peu orthodoxe comme étant de prime abord inhibée et non pas impulsive :

si nous prenons la pulsion en soi (Trieb in sich) - non pas dans l'impulsivité pulsionnelle où elle peut se libérer (nicht in dem Treiben, zu dem er ausgelöst werden kann), mais dans sa structure de pulsion (Triebstruktur) —, il apparaît alors que cette pulsion a précisément une tension interne et une charge contenue, une constriction et une inhibition qui requiert essentiellement d'être levée pour devenir une impulsivité pulsionnelle et pour pouvoir être ainsi effrénée au sens usuel du terme. (GA 29/30, 370)

Heidegger admet qu'il n'est pas tout à fait erroné de considérer les pulsions comme étant impulsives et de parler du «déchaînement des pulsions (Hemmungslosigkeit der Triebe) », mais ce n'est que parce que nous considérons seulement le résultat, ce à quoi elles poussent : prise en elle-même, la pulsion (Trieb) ne serait pas une impulsion (Drang). Afin d'être effrénée (Hemmungslos), afin d'être impulsive, la pulsion a besoin d'être activée par un déclencheur, un agent externe, un désinhibiteur. Puisque «la poussée par le pulsionnel caractérise [...] toute l'activité de l'animal » (347), il en découle que l'animal est en lui-même retenu, inhibé, pris en lui-même (Eingenommenheit in sich).

Si nous tentons de suivre Heidegger, cette inhibition primordiale de l'animal — son « être-pris en soi-même » — explique sa réceptivité, son excitabilité (Reizbarkeit), son irritabilité (Erregbarkeit) (372). L'inhibition rend possible la réceptivité particulière de l'animal parce que «l'excitabilité n'est possible que là où il y a désinhibition et encerclement» (373). Ce cercle d'excitabilité ou de stimulabilité explique pourquoi l'animal nous semble avoir un monde: «Cette compulsion [...] pousse et maintient l'animal dans une zone hors de laquelle il ne peut sauter et au sein de laquelle il y a quelque chose pour lui d'ouvert. » (364). L'animal est donc ontologiquement caractérisé par une certaine ouverture, mais d'une nature fondamentalement différente de celle de l'homme, puisque entre un monde de stimuli déclencheurs et un monde de 
choses significatives, il y a tout un monde, ou plus précisément, un abîme. L'abeille ne perçoit pas le miel comme miel, mais elle ne perçoit pas non plus le miel comme quelque chose d'autre, comme, par exemple, une chose-à-manger :

Dans toute son activité pulsionnelle, l'abeille est rapportée à l'endroit où se trouve la nourriture, au soleil, à la ruche, etc., mais ce rapport n'est pas une perception de ces choses en tant qu'endroit où se trouve la nourriture, en tant que soleil, etc. Dira-t-on que ces choses sont perçues comme étant quelque chose d'autre? Non, elles ne sont absolument pas perçues comme quelque chose, ni comme se trouvant être là [nicht als etwas und als vorhandsein)]. Il n'y a pas de perception, mais bien un comportement [kein Vernehmen, sondern ein Benehmen], une activité pulsionnelle [...]. (GA 29/30, 360-361; traduction modifiée)

Mais comment peut-il y avoir un comportement sans perception? Nous voyons que l'animal se dirige vers certaines choses et en évite d'autres, nous supposons alors qu'il peut fuir ou poursuivre et qu'il doit conséquemment avoir part à une forme de perception, de discrimination qui lui donne les choses qu'il poursuit et fuit d'une certaine manière, ne serait-ce que dans l'orée de l'agréable ou du désagréable. Or, là est précisément le point de l'énigmatique «caractère éliminant» du comportement : il n'y a chez l'animal qu'une modalité de comportement, en l'occurrence, la mise à l'écart.

\section{Le « comment » de l'ouverture animale : la privation du se-mouvoir}

La partie consacrée au «caractère éliminant»(Beseitigungscharakter) du comportement dans le $§ 60$ reste largement ignorée dans les études consacrées au cours, mais n’est rien d'autre que la pierre de touche (Krell 126) de toute l'ontologie heideggérienne de la vie. C'est cette analyse, curieusement, que laisse tomber Richir, sous prétexte qu'elle n'est pas «essentielle à la démonstration » et qu'elle n'est pas «tout à fait juste eu égard aux résultats de l'éthologie » (Richir 248). Et pourtant, il faut bien avouer que le «caractère éliminant» du comportement est 
de loin l'aspect le plus crucial de l'analyse heideggérienne de l'animalité, que Heidegger tient lui-même pour le «trait fondamental du comportement» (364). Le comment du comportement, c'est-à-dire la manière dont l'animal est en rapport avec autre que soi, s'y révèle enfin et s'y profile comme une dénégation de toute forme d'intentionnalité aux animaux.

À la différence du comportement humain — toujours intentionnel, c'est-à-dire toujours un «se-diriger-vers » (GA 20, § 5, 40) — «le comportement a comme caractéristique de mettre de côté » (GA 29/30, 363). Le « caractère éliminant » du comportement sera opposé à la capacité de l'homme de s'impliquer ou de laisser être (einlassen) l'étant (ce qui n'est rien d'autre que le laisser-retourner qui rend possible la totalité de tournures, comme nous le savons depuis Être et temps). En apparence, un animal se comporte envers un étant, il se dirige vers certaines choses, il s'oriente sur elles ou les recherche. En réalité, dira Heidegger, il n'y a jamais de mouvement vers... (Hin-zu) ou de conduite envers... : il n'y a que des comportements à l'encontre de..., des mouvements en contrecoup $(A b s t o \beta)$. L'animal se rapporte toujours à ce avec quoi il est en relation (que nous savons désormais être les désinhibiteurs) d'une manière telle qu'il les élimine, les écarte ou les met de côté («beseitigen» veut dire «faire disparaître », «supprimer » ou «anéantir»). «Non seulement le comportement montre-t-il qu'il ne s'implique jamais (sicheinlassen auf) dans quelque chose, mais il a même comme caractéristique de mettre de côté (beseitigen) ce avec quoi il est en relation. Ce trait fondamental du comportement (mettre de côté), cela peut être anéantir — dévorer —, mais cela peut être aussi éviter » (GA 29/30, 364). Heidegger déplore que non seulement nous n'ayons pas aperçu ce trait fondamental du comportement animal, mais que nous sommes même passés complètement à côté puisque nous allons généralement «jusqu'à mettre en œuvre une recherche de ce à quoi l'animal peut se rapporter » (365). C'est le cas, notamment, lorsque nous parlons du phototropisme des plantes 
qui tendent vers la lumière ou des animaux qui recherchent la lumière. Pour illustrer le caractère d'éradication ou d'élimination du comportement animal, Heidegger prend l'exemple du morio, un insecte qui fait partie de ces animaux qui «recherchent la lumière»: le morio, soutient catégoriquement Heidegger, ne recherche pas la lumière, «mais se comporte toujours en cela d'une telle façon que son comportement s'éloigne de l'ombre» (365). Il est fondamentalement erroné, dans l'esprit de Heidegger, de distinguer entre tropismes positifs (mouvement ou tendance vers) et tropismes négatifs (mouvements contre), puisqu'il n'y a jamais sous aucune forme de tendance vers... (Hin-zu), de recherche de quoi que soit: «Le comportement est toujours en soi une mise de côté » (364). Des êtres qui semblent attirés vers la lumière, nous devrions donc dire qu'ils fuient l'ombre plutôt que de dire qu'ils recherchent la lumière. Heidegger prend ici la peine de souligner l'impression d'arbitraire qui ne peut manquer de s'imposer: «Malgré tout, une impression d'arbitraire reste attachée à notre explication des choses. Se mouvoir vers la lumière, nous le saisissons comme s'il s'agissait de s'éloigner de l'obscurité, se mouvoir vers l'obscurité, comme s'il s'agissait de s'éloigner de la lumière. Pourquoi donc donnons-nous au fait de s'éloigner de... (Weg von...) la supériorité sur le fait de se mouvoir vers... (Hin zu...)? Pourquoi ne pas faire l'inverse?»(GA 29/30, 367). Cette interprétation est, dira sommairement Heidegger, «présumée plus intelligible » (367). Mais en quoi est-elle intelligible? Nous supposons généralement que la tendance à la fuite et à l'évitement n'est qu'une modalité du comportement parmi d'autres. C'est précisément pour cette raison que nous disons que les animaux se meuvent : parce qu'ils peuvent poursuivre ou fuir. Mais si «le comportement est toujours en soi une mise de côté » (364), cela signifie que la mise de côté n'est pas une modalité du comportement, une modalité parmi d'autres, mais que c'est en fait la seule possibilité. Un animal ne peut pas fuir ou poursuivre, il ne peut que fuir. 
Si nous gardons à l'esprit le rôle fondamental que joue la notion d'intentionnalité dans l'œuvre de Heidegger-l'être-dirigé-vers n'est pas seulement la structure de la perception, mais constitue la structure du comportement (Verhalten) et de l'expérience vécue (Erlebnis), l'essence même du «se sentir» et du «se mouvoir» (GA 20, §5, 40) - la fonction du Beseitigungscharakter du comportement animal apparaîtra évidente : il s'agit précisément de nier à l'animal toute forme d'être-dirigé-vers, d'être-hors-de-soi-auprès-de (qui n'est rien d'autre en fait que la structure du souci dans Sein und Zeit). Rigoureusement, donc, nous ne pouvons plus dire que les animaux se meuvent, puisque le mouvement pulsionnel des animaux n'a pas ce sens intentionnel d'«être-dirigé-vers...» qui fait partie de l'essence même du se-mouvoir : «le mouvement local est toujours le mouvement d'un être qui évite ou poursuit quelque chose » (Aristote, De Anima, III, 9, 432 b 25-30), toujours « un se mouvoir en direction de quelque chose ayant quelque importance pour la vie » (GA 22, 309 [326]).

\section{$X$. L'être-pris en soi-même ou la vie végétative de l'animal}

Malgré l'«impression de négatif»(GA 29/30, 367) manifeste que laisse cette interprétation du comportement animal, Heidegger insiste pour interpréter le mouvement vers la lumière (Hin zum Licht) comme s'il s'agissait d'un éloignement de l'obscurité (Weg vom Dunkel), affirmant que ce phénomène exprime quelque chose d'essentiel à l'animalité, notamment un certain contrecoup $(A b s t o \beta)$ dans le comportement : «C'est dans ce contrecoup que se manifeste l'être pris de l'animal en lui-même [In diesem Sich-abstossen bekundet sich die Eingenommenheit des Tieres in es selbst]»(367). Il a été fait peu état de cette inhibition primordiale de l'animal dans les analyses de l'ontologie heideggérienne de la vie, peut-être parce qu'on pense, à l'instar de Kacem, que le concept d'inhibition animale est «peu plausible ${ }^{16}$ ». 
Sans doute est-il inédit de caractériser l'animalité par l'inhibition, puisque cela est traditionnellement la marque de l'humanité, mais la caractérisation de l'animalité par l'inhibition est non seulement plausible, mais elle est aussi essentielle et nécessaire à la conceptualité de Heidegger : c'est elle qui permet d'instituer un abîme entre l'«être-hors-de-soi-auprès-de » de l'homme et l'«être-pris-en-lui-même » de l'animal.

Cette conception de l'animalité comme inhibition primordiale est réellement difficile à saisir, car elle va à l'exact opposé de la conception commune de l'animalité : être inhibé, c'est être empêché, refoulé, paralysé, impotent. Dans le concept de Benommenheit s'entend une «torpeur » qui est le plus souvent caractéristique de notre conception de la vie végétative plutôt qu'animale. Mais pour suivre Heidegger, il faut cesser de penser l'animal en différenciation avec le végétal, puisqu'il s'agit là d'un seul et même mode d'être : celui du vivant, du seulement vivant (Nur-noch-Leben). En concevant l'animal comme étant primordialement inhibé, pris en lui-même, Heidegger renverse un axiome fondamental de la conception hégélienne de l'humanité comme inhibition en soi de l'animalité et, en vérité, il détruit complètement notre conception usuelle de l'animalité. Traditionnellement, les animaux sont caractérisés par des tendances (Hin$z u$ ) et des impulsions (Drang) effrénées qui les poussent à agir de telle ou telle manière. Souvent même, le terme d'animalité est synonyme d'impulsivité en ce qu'il fait référence à une force active, une force vitale ou irrationnelle, à une puissance déchaînée, à un surplus d'énergieénergie bouillant également à l'intérieur des hommes, mais que l'humanité serait parvenue à maîtriser, à nier, à sublimer, à transcender. Le plus souvent nous imaginons que les animaux sont précisément en manque d'inhibition (Hemmungslos), complètement privés de retenue et qu'ils sont incapables de ne pas laisser libre cours à leurs pulsions, alors que nous, êtres humains, nous sommes capables de les maîtriser, de les réfréner, de les bloquer, voire de les sublimer. L'homme 
ne se distingue pas des bêtes parce qu'il dispose d'auto-mouvement, remarquait Hegel—fidèle ici à toute la tradition philosophique aussi bien qu'au sens commun-, «mais parce qu'il est capable d'inhiber le mouvement et de briser par là son immédiateté et sa naturalité » (Hegel 7778). $\mathrm{Si}$, pour suivre Heidegger, il faut rompre, comme le remarque Dastur, « avec l'idée courante qui oppose pulsion et inhibition » et ainsi voir que «la structure de la pulsion exige d'une façon essentielle la dés-inhibition » (Dastur 56), il faut du même coup réviser complètement le concept même d'animal : les animaux ne sont pas des êtres spontanément actifs, qui rencontrent des entraves et des résistances (Hemmungen), mais des êtres de prime abord inhibés qui rencontrent des désinhibiteurs (Enthemmungen), des déclencheurs de pulsion.

Heidegger rend tout à fait plausible qu'un être pulsionnellement en rapport avec des désinhibiteurs ne se tienne pas en vis-à-vis d'un monde, car le désinhibiteur n'a rien d'une chose ou d'un «quelque chose » qui pourrait survivre et rester là pour l'animal au-delà de l'excitation provoquée (GA 29/30, 372). Mais si la «chose excitante» ne persiste pas, que devient alors l'animal? La question vaut tout de même la peine d'être posée! Qu'arriverait-il si l'animal ne rencontrait jamais de désinhibiteurs? S'ennuierait-il? Imaginez un animal dans un état complet d'isolement, dans un environnement tel que justement aucun stimulus déclencheur n'est susceptible de déchaîner ses pulsions. Que se passerait-il? Heidegger ne considère pas une telle possibilité, mais étant donné les indications qu'il nous a déjà données, une seule solution semble possible : l'animal tomberait dans un état de dormance, de profonde léthargie puisque, tel que nous le présente Heidegger, l'animal n'a simplement aucun pouvoir-être par-delà ce qui lui est permis par ses désinhibiteurs. Sans eux, il n'est rien, il n'est pas animé, au sens effectif du terme, il tombe, en quelque sorte, en hibernation : «L'animal s'endort dès que rien ne le sollicite » (Caron 691). Entre deux stimuli, l'animal végète, il sombre dans une espèce de coma jusqu'à ce 
qu'un stimulus vienne à nouveau l'éveiller de sa torpeur. À l'inverse, parce qu'il est caractérisé par la possibilité, le pouvoir-être et non, comme l'animal, par la simple capacité ${ }^{17}$, le Dasein humain, lorsque l'étant ne le stimule plus, ne l'excite plus, ne lui dit plus rien, s'ennuie, il s'ennuie profondément, comme Heidegger l'a longuement illustré dans la première partie du cours. La capacité ou l'aptitude (Fähigkeit)—qui «domine complètement le mode d'être de l'animal » (GA 29/30, 370)—n'est pas une force active, une spontanéité, une impulsivité, une puissance d'agir, c'est plutôt une puissance de pâtir, une réceptivité, un être-excitable, une capacité d'être «affecté », au sens d'être excité et stimulé (déjà, remarquons-le, le sens donné à l'«affection» des animaux dans $\hat{E} t r e$ et temps). La déconstruction de l'idée d'impulsivité animale—par le détour de la conception de la pulsion animale comme étant d'emblée inhibéeest en ce sens éminemment significative en ce qu'elle place l'animal du côté de la pure et simple capacité et jamais du côté de la possibilité (du pouvoir-être) : l'animal est par là situé de l'autre côté de l'abîme ontologique.

\section{L'impulsion comme souci non libre : la genèse du monde, de la finitude, de l'individuation}

Dans un séminaire donné au semestre d'été 1928, Heidegger distingue longuement deux formes de possibilité en montrant la différence essentielle qui existe entre le concept de force active (la vis activa de Leibniz) et le concept de capacité ou de faculté (la potentia activa des scolastiques). Ce dernier renvoie à une simple capacité qui n'a pas à être en acte, à un pouvoir qui peut (ou peut ne pas) s'actualiser selon les circonstances extérieures, car il a «besoin d'une excitation externe ou d'un stimulus ${ }^{18}{ } »$. À l'inverse, le concept leibnizien de vis activa (force active) est davantage qu'une simple prédisposition à agir, car elle est en elle-même im-pulsion, 
elle «pousse à », elle «tend vers » $(H i n-z u)(G A$ 26, 101-103 [82]). D'elle-même, l'impulsion se pousse et mène à l'activité sans qu'il y ait besoin d'un impetus, d'un stimulus (stimulo), d'une excitation externe pour être en acte : «drive (Drang) is as such already released» (101-103 [82]). À la différence du Trieb animal qui nécessite l'activité d'un déclencheur externe, l'impulsion (Drang) est d'emblée désinhibée, d'emblée effrénée : elle est « une " tendance” (Hin zu) qui apporte elle-même avec soi son moteur» (SZ 195 [149]). Un être caractérisé par l'impulsion (Drang) rencontre des inhibiteurs ou des entraves (Hemmungen) à ses impulsions et non pas des excitants et des désinhibiteurs (Enthemmungen) : il reçoit de l'extérieur non pas sa force d'agir, mais un empêchement d'agir. Or, cette rencontre de l'entrave et de la résistance fait apparaître des «êtres individuels », disait Schelling, fait naître «ce sentiment confus de l'individualité » dont parle Leibniz. L'impulsion, dans sa poussée hors d'elle-même, rencontre des oppositions, des entraves ou des inhibiteurs, c'est-à-dire qu'elle est limitée, restreinte, entravée, empêchée. «Formellement, finitude veut dire : restriction, limitation » (GA 26, 116117 [94]). La finitude, affirme Heidegger, doit être définie comme une composante de l'impulsion (Drang): chaque impulsion doit rencontrer du résistant qui s'oppose à son mouvement. Pourquoi? Parce que, d'elle-même, l'impulsion peut être-tout : «Because drive (Drang) can be the whole universe potentially but in fact is not, drive is for that reason related to resistance in its driving »(121-122 [98]). Dans cette expérience du «contre » et de l'opposition, la monade s'inhibe, retourne en elle-même, elle s'individualise. La rencontre d'entraves ou d'inhibiteurs (Hemmungen) la pousse à retourner en elle-même (re-flexio). S'individualisant, la monade devient un mundus concentratus, un monde concentré, un microcosme. La structure de l'impulsion est d'être configuratrice d'unité et en unifiant le divers, elle anticipe (112-113 [90]) : «From drive (Drang) itself arises time » (114-115 [92]). Dans le Drang, il y a donc déjà genèse 
du monde, de la finitude, de l'individuation - ce qui deviendra, l'année suivante, les trois concepts fondamentaux de la métaphysique.

Ce n'est pas parce qu'il ne peut se représenter une réalité objective que l'animal n'a pas de monde, mais bien parce que lui est retiré ce pouvoir décisif de la rencontre de la résistance. Dans sa critique de la réalité conçue comme objectivité, Heidegger s'appuie principalement sur la percée de Dilthey qui a mené à identifier la résistivité comme «caractérisation phénoménologique de la réalité du réel» (SZ 209 [157]). La réalité n'est pas donnée «primairement à la pensée et à la saisie », mais elle est immédiatement expérimentée dans l'impulsion et la volonté comme résistance. Or, si les animaux sont ontologiquement caractérisés par l'impulsion (Drang), ils sont bel et bien initiés à cette expérience primitive de la réalité qui naît de la rencontre de la résistance, même s'ils sont incapables d'élever ces «centres de résistance » à la condition d'objets et de les saisir dans leur «être-tel» (Sosein), comme le pensait Scheler (Scheler, 54). Les bêtes vivraient alors dans un sentiment diffus et confus de réalité, mais il y aurait tout de même là une forme de conscience phénoménale, puisque la conscience apparaît déjà là où il y a cette re-flexio primitive qui naît de la rencontre de résistances.

Privé d'impulsivité, l'animal n'a plus part à ce sentiment primitif de réalité parce qu'il ne rencontre pas des entraves à ses impulsions, mais des désinhibiteurs de pulsion. C'est ce geste qui permet de priver définitivement l'animal de monde. Si nous l'avons si peu remarqué, c'est sans doute parce que le rôle fondamental joué par l'impulsion (Drang) dans la pensée de Heidegger est passé largement inaperçu dans les études heideggériennes. Et pourtant, ce désintérêt pour le concept de Drang est difficile à comprendre, puisque c'est bien au moyen des phénomènes de la tendance et de l'impulsion (Hang und Drang) que Heidegger expose le versant 
inauthentique du souci (Uneigentlich Sorge) au $§ 41$ d'Etre et temps! Succombant à «l'impulsion “à vivre" » (Drang zu «leben »), au «penchant à "se laisser porter" par le monde ("vivre") » (SZ 196 [149]), le Dasein se laisse «entraîner par ce à quoi le penchant aspire » (SZ 195 [149]) et se laisse pré-donner ou imposer ses possibilités par l'étant auprès duquel il se trouve, de sorte que «dans la pure impulsion, le souci n'est pas encore devenu libre (SZ 196 [150]). En n'excluant pas que ces phénomènes du Hang und Drang qui sont à la racine de la Verfallenheit du Dasein « constituent aussi ontologiquement l'étant qui "vit" sans plus » (SZ 195 [194]), il devient dès lors bien difficile de comprendre cette Drang «zu leben » qui mène à la ruine de soi-même (Ruinanz) autrement que comme étant le fait d'une animalité en lui.

Puisque «l'impulsion est le souci qui n'est pas encore devenu libre (Drang ist die Sorge noch nicht frei geworden)»(GA 20, 411 [248]), on comprend pourquoi Heidegger se devait finalement de reconnaître que «la vie doit être comprise comme un mode d'être auquel appartient un être-au-monde (Leben muss verstanden werden als eine Seinsart, zu der ein In-derWelt-sein gehört)» (SZ 246 [182]), que l'animal est « un étant auquel nous devons aussi attribuer (d'un point de vue formel) le mode d'être du Dasein » (GA 20, 223 [242]). Ce n'est qu'une fois que nous avons pris la mesure du rôle central joué par le concept de Drang chez Heidegger que le geste (de prime abord incompréhensible) de priver les animaux de toute forme d'impulsivité prend son sens véritable : que la pulsion (Trieb) soit de prime abord inhibée, qu'elle ne soit pas d'elle-même une impulsion (Drang), coupe court à tout rapprochement entre l'animalité et l'inauthenticité du Dasein ${ }^{19}$.

En pensant l'animalité comme inhibition primordiale, Heidegger règle le problème que lui posait la constitution ontologique des animaux dans Être et temps, mais il prive en même temps les animaux de cette spontanéité que nous leur avons toujours reconnue. L'animal « habite 
son possible ${ }^{20} »$, c'est-à-dire que «la possibilité de l'animal correspond à sa réalité effective et réciproquement» (Schelling 255). Ce qui ne veut en fait rien dire d'autre que ceci : l'animal n'a pas de pouvoir-être excédent ce qu'il est. Dans la déconstruction heideggérienne de la pulsion (Trieb) comme impulsion (Drang), ce n'est rien d'autre que la spontanéité animale qui s'évanouit.

\section{La déconstruction de l'animalité comme réduction biologique de l'animal}

Au terme du cours de 1929-1930, la voie de l'ontologie heideggérienne de la vie annoncée dans Être et temps comme une «interprétation privative » se révèle être une réduction au biologique, une rature de tout ce qui a trait à l'existence humaine, au biographique. L'argumentaire des Concepts fondamentaux de la métaphysique n'est intelligible que si nous considérons que, pour Heidegger, la vie animale n'est pas essentiellement distincte de la vie végétale : la « vie », dira Heidegger en 1929-1930, «c'est-à-dire la manière d'être de l'animal et de la plante» (281). Malgré que Heidegger prétende avoir laissé dans Être et temps la question de l'être des animaux et des vivants «ontologiquement indéterminée » (SZ 50 [59]), on remarque, à la lueur de l'ontologie de la vie, qu'y étaient en vérité déjà posés les jalons principaux qui nous mènent à la réduction biologique de la manière d'être des animaux à laquelle nous avons assistée. En effet, comment peut-on penser que la question de l'animal est complètement laissée en suspens comme «problème à part » dès lors que la sensibilité animale est nommée autrement et réduite à excitation (Reiz) et stimulation (Rürhung) des sens (SZ 242 [346])? Ou, plus généralement, dès lors que les animaux sont déjà nommés « seulement vivants » (Nur-Lebenden) (SZ 346 [242]), «rien de plus que vie»(Nur-noch-leben) (50 [59])? La méthodologie ou la «stratégie», si l'on ose dire, de cette analytique de la vie est somme toute 
simple: elle consiste à trouver des équivalents fonctionnels pour tout ce qui concerne les animaux qui a de près ou de loin à voir avec nous-mêmes : ainsi, l'animal n'est pas affecté, mais «stimulé »; il voit, mais ne regarde pas (GA 54, 158-9); il a des organes de préhension, mais pas de mains $(\mathrm{GA} 8,18)^{21}$; il ne meurt pas, mais périt; il « ne mange pas, il avale sa nourriture » (GA 29/30, 310); etc.

Tout au long, comme on le lui reprochera ad nauseam, Heidegger tient délibérément à l'écart les animaux dits «supérieurs », c'est-à-dire précisément ceux que nous considérons habituellement comme les animaux. Le problème n'est pas que les animaux qui «affichent dans une correspondance apparemment fidèle à notre tenue de rapport» (351) soient mis méthodologiquement de côté, mais bien qu'ils ne reviennent pas atténuer ou relativiser la radicalité et la portée des analyses qui ne visent rien de moins qu'à élucider l'essence de l'animalité! De là la réception embarrassée du cours : de quoi avons-nous donc traité tout au long de l'ontologie de la vie animale de 29/30? De la vie. Pourtant la vie, remarquait déjà Aristote, n'est pas le «trait définitionnel » de l'animal, mais la sensibilité, la perception sensible : «Quant à l'animal, c'est la sensation ou la perception (aisthesis) qui est à la base de son organisation : même, en effet, les êtres qui ne se meuvent pas et qui ne se déplacent pas, du moment qu'ils possèdent la sensation, nous les appelons des animaux (zôa) et non plus seulement des vivants (ou zên monon)» (Aristote, De l'âme, II, 413b 1-4). Même la critique derridienne ne va pas assez loin : non seulement Heidegger parle-t-il de l'animal au «singulier général » comme s'il n'y en avait qu'un, mais la thèse heideggérienne va en réalité bien plus loin que cela, car il ne saurait y avoir pour Heidegger de différence ontologiquement décisive entre un animal et un végétal. Qu'il s'agisse de rhododendrons, de carottes, de lézards, de chiens ou de chimpanzés, tous ces étants seront dits « seulement vivants ». La distinction entre une vie végétative ou une 
vie organique (digestion, circulation, respiration, etc.) et une vie animale ou une vie de relation (qui se sent, se meut, perçoit et désire) est étrangère à l'ontologie heideggérienne de la vie.

Ontologiquement, l'animalité n'est pas, pour Heidegger, distincte de la végétalité. La vie, c'est essentiellement le biologique : «le fonctionnement du cœur chez l'animal n'est pas un processus autre que le fait de prendre ou de voir » (349). Les comportements des animaux ne sont pas essentiellement différents de la croissance des végétaux et du fonctionnement des organes vitaux : «entendre, voir, prendre, chasser, s'enfuir, dévorer, digérer, etc. — bref tous les processus organiques » (349). Et c'est uniquement parce que Heidegger pense les comportements animaux sur le modèle des mouvements tropistiques des végétaux qu'il peut passer sous silence le problème de la relation au temps des animaux, puisque par définition les tropismes sont indifférents à la situation, c'est-à-dire à ce qui venait avant l'excitation et à ce qui viendra après. Ce qui les caractérise, c'est leur indifférence au temps. Un être purement tropistique ou «taxique » - nous pourrions maintenant dire : purement instinctif — ne peut absolument rien apprendre ni rien anticiper: il est rigoureusement le représentant de son espèce et n'a pas d'histoire individuelle. Gadamer a très bien saisi le sens des analyses de Heidegger lorsqu'il rappelle que celui-ci nous a appris «que l'être propre de l'animal n'est pas celui d'un Da-sein particulier, mais celui de l'espèce, que c'est l'espèce qui est "là" pour l'animal » (Gadamer 80). Cela est bien aisé à soutenir dès lors que la question du déploiement temporel propre aux animaux (ce que Heidegger nomme la Bewegheit, c'est-à-dire le fait qu'un animal naisse, parvienne à maturité, vieillisse et, finalement, meure) «a été délibérément tenue à l'écart » (GA 29/30, 386). La question du rapport au temps des animaux a été tenue délibérément à l'écart parce que «naissance, maturation, vieillissement, mort, tout cela rappelle trop l'être de l'homme, que nous connaissons comme être historique » (385; nous soulignons). À cet égard, Heidegger 
déplore qu'on soit «même allé jusqu'à parler des organismes comme d'êtres ayant une histoire » (385) : «Tout comme il reste suspect de parler de l'animal comme d'un être historique ou même d'un être qui a son histoire, la question se pose de savoir si la mort de l'animal et la mort de l'homme est la même mort, même si des concordances physico-chimiques et physiologiques peuvent se constater »(GA 29/30, 387). Après la lecture du cours de 1929-1930, nous sommes mieux en mesure de comprendre cette amortalité des bêtes : ontologiquement indistincts des végétaux, prisonniers dans la sphère du biologique, les animaux ne participent en rien à quelque chose comme une vie biographique, une histoire personnelle en regard de laquelle seulement la venue de la mort prend son sens véritable. Que, dans Être et temps, Heidegger prive les animaux de la possibilité de mourir, ne leur reconnaissant qu'un «périr », une «mort physiologique ou biologique » (SZ 247 [182]) illustrée bien à propos avec l'exemple d'un fruit venant à maturité (SZ 243 [181]), cela a alimenté bien des querelles dans les études heideggériennes, et pourtant, ce qui est le plus incompréhensible pour nous ce n'est pas que Heidegger ait pu nier la possibilité de mourir aux animaux, mais bien plutôt qu'il ne leur ait jamais reconnu une vie véritable, mais seulement une vie mutilée et réduite à une expression dans laquelle elle ne se distingue pas d'une vie végétale ou végétative.

Nous voyons toute la méprise qu'il y a à considérer que la conception heideggérienne de l'animalité trahit son appartenance à la tradition métaphysique : elle est tout sauf traditionnelle. La déconstruction de l'animalité qui a lieu dans les Concepts fondamentaux de la métaphysique est à ce point radicale qu'elle est, en réalité, une pure et simple destruction de l'animalité en ce qu'elle fait disparaître l'idée même d'une vie animale, d'une vie propre aux animaux: la tripartition aristotélicienne des âmes (végétative, animale, rationnelle) laisse place à un dualisme entre le biologique (la zoé) et le biographique (le bios) ${ }^{22}$. La vie de l'animal n'est jamais une vie : 
l'animal est «en vie», certes, mais il ne vit pas réellement quelque chose, il n'éprouve rien, il végète, au même titre que le «seulement vivant». Ce que nous interprétons depuis Aristote comme constituant le propre des animaux, c'est-à-dire la vie de relation, le percevoir, le «se sentir », le «se mouvoir » et même cette forme de désir irrationnel qu'est l'impulsion, se révèle, en définitive, proprement humain. La sensibilité et la mobilité des animaux ne sont pas essentiellement différentes de celles que nous retrouvons chez les végétaux : il s'agit d'une forme d'excitabilité des sens et d'une forme de mobilité forcée (l'animal ne peut plus fuir ou poursuivre — les deux mouvements fondamentaux du désir chez Aristote — il ne peut que fuir).

Ce rapt du propre des bêtes est, comme nous avons tenté de le montrer, nécessaire à la cohérence de la pensée heideggérienne puisque, simplement inintelligent et aphasique, simplement irrationnel, l'animal vivrait néanmoins dans l'éclaircie de l'être! En définitive, si nous avons l'exigence de dépasser la conception traditionnelle de l'homme comme animal rationnel (GA 29/30, 101; SZ, 183), ce n'est pas tant parce qu'elle insiste trop sur la rationalité de l'homme, mais c'est plutôt parce qu'elle ne spécifie pas bien l'essence de la différence zooanthropologique. En pensant l'animal comme alogon, nous n'allons pas assez loin, car cette privation est consécutive d'une indigence plus essentielle : la privation de la perception véritable, c'est-à-dire de cette perception qui rassemble et unit grâce au als et qui fonde la «manifesteté anté-prédicative » (490), «l'ouverture prélogique à l'étant» (494). Pour Heidegger, c'est parce que l'animal est privé de monde—c'est-à-dire tout accès à l'«en tant que »— que le logos lui est refusé et non l'inverse : la tradition a toujours pris les choses à l'envers en privant d'abord l'animal du $\log _{o s}{ }^{23}$.

L'erreur du sens commun et de la tradition philosophique qui mène à méconnaître l'essence de l'homme repose sur la conviction que les animaux font l'expérience de l'étant, que 
celui-ci leur est manifeste en un sens ou un autre. Elle repose finalement sur notre croyance que les animaux sont «là » eux aussi au milieu des choses, que chacun d'eux est le centre à partir duquel s'articule un monde ambiant. Or, penser avec Rilke que «l'animal est dans le monde, alors que nous nous tenons devant lui, du fait de la singulière tournure qu'a prise notre conscience » est, aux yeux de Heidegger, «une des dernières conséquences de l'oubli de l'être ${ }^{24} \gg$ (Parménide, GA 54, § 8, 229). Tant et aussi longtemps que nous penserons, bêtement, que les animaux perçoivent réellement et font réellement quelque chose, qu'ils ne sont pas seulement «en vie », mais qu'ils vivent à proprement parler, jamais nous ne comprendrons «cette solidarité de l'être (de l'étant) et de l'être de l'homme» qui nous rend les dieux plus proches que les animaux ${ }^{25}$. Mais il faudra, espérons-le, beaucoup plus que la promesse de l'éveil de la question de l'être pour nous forcer à croire que les animaux sont une «réalité impénétrable» et que tous les sons qu'ils émettent sont de «simples bruits», des bruits insignifiants ${ }^{26}$.

\section{Notes}

${ }^{1}$ «La vie est un mode d'être spécifique (eine eigene Seinsart), mais il n'est essentiellement accessible que dans le Dasein. L'ontologie de la vie s'accomplit sur la voie d'une interprétation privative (privativen Interpretation); elle détermine ce qui doit être pour que puisse être quelque chose qui ne serait «plus que vie» (Nur-noch-leben). La vie n'est pas un pur être-sous-la-main, ni, encore, un Dasein. Et le Dasein, inversement, ne peut en aucun cas être déterminé en affirmant qu'il est vie (ontologiquement indéterminée), plus quelque chose d'autre (als leben - (ontologisch unbestimmt) und als überdies noch etwas anderes) » (SZ, § $9,50[59])$.

${ }^{2}$ Concepts fondamentaux de la métaphysique : monde, finitude, solitude (individuation) [par la suite GA 29/30], 266. Les éditeurs ont interchangé «Vereinzelung » pour «Einsamkeit».

3 « The animal does not experience being as being, does not have access to beings as such. Here and throughout the final hours of the lecture course Heidegger appeals to the animal's lack of apophansis, its lack of logos, as the secret of its benumbed behavior in an 
impoverished world. Not the foundational hermeneutical-as but the derivative apophantis-as comes to dominate - and undo fundamental ontology » (Krell 129)

4 «Tout vivant, dans la mesure où il est, a un monde, ce qui ne vaut pas pour le non-vivant. Tout vivant s'oriente sur quelque chose, il se dirige vers lui, l'évite, etc. à vrai dire tout cela de manière encore indistincte. On ne peut interpréter de la sorte les protozoaires et les êtres vivants en général que de façon indirecte, par analogie avec nous. Mais en même temps que cet être découvre un monde, son être lui-même se découvre. Cet étant sait de quoi il retourne avec lui-même, quoique seulement en un sens très vague et très général » (Prolégomènes au concept de temps [par la suite GA 22], 207-208 [227-228]).

5 «Tout être vivant a un monde ambiant, non comme quelque chose de disponible à côté de lui, mais qui lui est ouvert, qui est là, à découvert. Ce monde peut être très simple (pour un animal primitif). Mais la vie et son monde ne sont jamais deux choses juxtaposées comme deux chaises côte à côte, la vie "a" au contraire son monde. [...] [L]'essentiel est manqué si je ne vois pas que l'animal a un monde [das Tier eine Welt hat]. De même, nous sommes également toujours dans un monde, de telle sorte qu'il est ouvert. Un objet, par exemple une chaise, est simplement disponible. Mais toute vie est là de telle sorte qu'un monde est également là pour elle [Alles Leben aber ist so da, dass für es eine Welt mit da ist] » (Heidegger, Conférences de Cassel 179).

${ }^{6}$ À partir de 1935, Heidegger ne parle jamais plus de «pauvreté » ou de «privation » en monde, mais affirme simplement que les animaux n'ont pas de monde. Par exemple, dans l'Introduction à la métaphysique : «L'animal n'a pas de monde (Welt) ni non plus de monde environnant (Umwelt). Le monde est toujours monde spirituel »(GA 40, 54). Dans les Beiträge, il reconnaîtra explicitement que c'était une erreur de définir les animaux par la pauvreté en monde, même pensée comme privation en monde, parce qu'il sont simplement sans monde (GA 65, § 154).

7 Houillon, « Pauvrement habite l'animal... », 123. Cette interprétation ne manquera pas d'étonner, en ce que Houillon reconnaît lui-même par ailleurs que l'animal vit hors sens et hors possibilité : «L'habitation, qui est l'habitation du sens, n'est pas une possibilité de l'animal, qui n'est même pas hors sens puisque le sens n'est pas une de ses possibilités ou que l'animal n'a pas de possibilité » (117). Pourtant, se rapporter à l'étant comme ceci ou cela, c'est bien vivre immergé dans le sens!

${ }^{8}$ « They animal's world lacks "what-is", though of course it is full of all sorts of things which animals eat, climb, walk on, live in, play with, and so on. Animals establish all these relationship to things without any understanding of the being of what-is ». «Perhaps, this is unfair to animals. Heidegger usually takes the position that animals do not have any understanding of being or language, or any "world" in his technical sense. At other places, however, he remarks that while plants are word-less, "animals are "world-poor", suggesting that animals do have some sort of minimal context of significance. Animals may have a 
rudimentary network of significance grounded in their "practices" (or ours), but they do not have language which give them an understanding of being » (White, Time and Death 2-3, 7n).

${ }^{9}$ Derrida, L'animal que donc je suis 218-219 : «L'animal, ne sait pas "laisser être", "laisser être la chose telle qu'elle est'. Il a toujours un rapport d'utilité, de mise en perspective, il ne laisse pas la chose être ce qu'elle est, apparaître comme telle sans un projet guidé par un "tuyau" étroit de pulsions, de désirs. Une des questions qui se poserait alors serait de savoir si l'homme le fait. [...] Car c'est cela le rapport de l'étant en tant que tel, c'est-à-dire le rapport à ce qui est en tant qu'on le laisse-être ce qu'il est c'est-à-dire où on ne l'approche pas, où on ne l'appréhende pas à partir de notre propre perspective, de notre propre dessein. Pour avoir rapport au soleil en tant que soleil, il faut que, d'une certaine manière, j'aie rapport au soleil tel qu'il est en mon absence et c'est en effet comme cela que se constitue l'objectivité, à partir de la mort. »

${ }^{10}$ Derrida a sans aucun doute orienté de la manière la plus décisive la réception du cours : «toute la déconstruction de l'ontologie, telle qu'elle s'engage dans Sein und Zeit et en tant qu'elle démet, en quelque sorte, le spiritus cartésiano-hégélien dans l'analytique existentiale, se trouve ici menacée dans son ordre, sa mise en œuvre, son dispositif conceptuel par ce qui s'appelle si obscurément encore l'animal » (Derrida, De l'esprit, 71). Krell abonde dans le même sens : "When Heidegger tries to separate Dasein from the animal, or to dig an abyss of essence between them, he causes the whole of his project to collapse back into the congealed categories and oblivious decisions of ontotheology. » (Krell 105).

11 «Séminaire du Thor», dans Questions IV, 150 : «Le Verfallen, entendu ontologiquement, est la naturalité même du Dasein tel qu'il ne peut s'occuper des choses qu'en s'occupant de l'être. Mais s'occuper de l'étant n'est possible et compréhensible que par le déval à partir de l'être. S'il est nécessaire que l'être, dans la vie humaine, demeure athématique, si, en d'autres termes, le but de Etre et temps n'est pas de ramener le Dasein quotidien à une thématisation de l'être qui ne constitue pas son propre, il n'en reste pas moins que la "vie humaine" ne serait pas possible en tant que telle sans l'éclaircissement préalable et insu de l'être. Tel est le sens des analyses célèbres et pourtant méconnues de l'ustensilité dans Être et temps. Le caractère ustensilié des objets n'a pas besoin de devenir thématique, et pourtant c'est sur la chaise en tant que telle que je suis assis. »

12 «Ce qu'est et comment se détermine la phusis », dans Questions II, 521 : «Par cette capacité critique de distinguer (cette prévision), l'homme est tiré hors du simple engourdissement dans ce qui le harcèle et le préoccupe, tiré et placé dans la relation à l'être; il devient [...] ex-sistant, il ex-siste, au lieu de simplement vivre et d'attraper au vol la "réalité", grâce à sa proximitié vitale. »

13 «L'aisthesis de l'homme est bien plutôt dès le commencement quelque chose d'autre que la nature, bien que l'entente de l'être où elle se trouve insérée ne soit pas encore éveillée, soit indéterminée et reste de prime abord indéployée. [...]. Cette indétermination de l'entente de l'être (chez le nouveau-né) ne signifie par rien, mais est quelque chose de positif, - qui 
manque à l'animal pendant toute sa vie parce que l'entente de l'être, d'une façon générale, lui fait défaut »(GA 34, 235-236 [264]). Voir aussi le cours sur Parménide : " "Les animaux nous voient" dit-on. Mais les animaux ne regardent pas. Jamais "épier", "être aux aguets", "fixer". "ouvrir un oeil de taureau" chez l'animal ne donne un se-découvrir de l'être; jamais l'animal dans son prétendu regard ne se porte lui-même à l'émergence en découvrant en même temps que lui un autre étant » (GA 54, 158-159; tr. Haar, 78).

${ }^{14}$ C'est tout le sens de la curieuse expérimentation que relate Heidegger à propos d'une abeille placée devant un bol de miel, dont on sectionne l'abdomen : l'abeille «continue tranquillement à boire tandis que le miel ne cesse de s'écouler derrière elle »: «Elle ne constate ni la surabondance de miel, ni même la disparition de son abdomen - ce qui est encore moins compréhensible » (353). Heidegger conclut : « l'abeille est simplement prise par la nourriture » et «cette emprise dans la poussée exclut la possibilité de constater une présence » (354). L'abeille mutilée s'empiffre inlassablement parce que l'inhibition par saturation ne peut se produire.

15 Ici, les choses se compliquent considérablement : cette poussée directionnelle ressemble fort à une orientation dans l'espace. L'abeille retournant à la ruche ne retrouve-t-elle pas son chemin? Et le chien perdu « qui retrouve son chemin grâce à son flair » (356) n'ouvre-t-il pas quelque chose comme une spatialité? N'y a-t-il pas là un rapport à l'espace? Et, du même coup, un rapport au temps, puisqu'il doit bien y avoir quelque chose comme de la mémoire et de la reconnaissance qui permettent aux animaux de s'orienter et de retrouver leur chemin? Heidegger se fera en cette matière bien avare : il tentera de montrer que dans ce mouvement de retour à la ruche, l'abeille ne s'oriente pas vraiment, car « il n'y a orientation que là où l'espace est ouvert en tant que tel, et où il est possible de distinguer des régions ainsi que des lieux repérables au sein de celles-ci » (355).

Heidegger insiste sur l'idée que l'abeille s'oriente grâce à une pulsion directionnelle et non pas grâce à des « marques indicatrices » (Orientierungsmarken) (357), des signes ou des points de repères. Et pourtant, il avouera lui-même que quelque chose de tel peut néanmoins jouer un certain rôle du fait que la pulsion directionnelle cesse « aussitôt que la possibilité de rentrer au lieu d'origine est assurée par les alentours reconnus de la ruche » (360; nous soulignons) et qu'elle n'entre tout simplement pas en jeu si la ruche ne se trouve pas sur un terrain désert: "Là où, par contre, les alentours sont recouverts d'arbres et de maisons, l'abeille suit ces arbres et ces maisons comme des jalonnements (Wegmarken), et elle ne s'abandonne pas à la pulsion directionnelle » (359). Fort malheureusement, Heidegger n'explicite pas plus longuement le rôle joué par ces énigmatiques Wegmarken et Orientierungsmarken, ce qui aurait pourtant mené à reconnaître qu'il y a aussi pour l'animal une forme de phénoménalité et qu'il doit y avoir quelque chose comme du sens et de la signification. Et surtout, cela aurait permis d'ouvrir la question du rapport au temps des animaux, puisqu'il y a bien, dans cette reconnaissance de l'abeille qui s'oriente sur les «alentours reconnus » et ne se laisse plus aller à la poussée directionnelle, une forme de mémoire et d'anticipation. 
${ }^{16}$ Kacem, 131 : «L'animal pour être ainsi désinhibé par ce qui l'entoure, devrait être inhibé au départ; ce qui est peu plausible, dans les termes mêmes où Heidegger nous l'expose ».

17 «Par pouvoir, nous entendons toujours la possibilité d'une tenue de rapport à... [...]. À la différence de ceci, nous avons appelé aptitude la possibilité de se comporter, d'être rapporté en étant pris et emport. » (GA 29/30, 485); «L'aptitude domine complètement le mode d'être de l'animal » (370). Sur la distinction entre l'être-apte de l'animal et l'être-apprêté-pour de l'outil, voir la distinction entre l'organisme et la machine : sections $\S 51$ à $§ 57$.

${ }^{18}$ Heidegger, Metaphysical Foundations of Logic 82 (par la suite GA 26).

${ }^{19}$ Rapprochement qui soutient de bout de bout la lecture d'Agamben dans De l'ouvert : « Le Dasein est simplement un animal qui a appris à s'ennuyer, qui réveillé de sa propre stupeur (Benommenheit) à sa propre stupeur (Benommenheit) »; "Dans l'expérience de l'ennui profond, l'homme a pris le risque de suspendre son rapport de vivant avec son milieu » (107).

${ }^{20}$ «Le bouleau ne dépasse jamais la ligne de son possible. Le peuple des abeilles habite dans son possible. La volonté seule [...] force la terre à sortir du cercle de son possible» (« Dépassement de la métaphysique », dans Essais et conférences 113).

21 «Les singes aussi ont des organes de préhension, mais ils n'ont pas de mains. La main est séparée de tous les organes de préhension - les pattes, les ongles et les griffes — infiniment, c'est-à-dire qui pense, peut avoir une main et accomplir dans un maniement le travail de la main » (Qu'appelle-t-on penser?, 90)

22 « Ni la plante, ni l'animal quoique déterminés comme zoè, n'ont aucun bios, aucune vie au sens d'une biographie » (Heidegger, Aristote, Métaphysique, $\theta$ 1-3, 127).

23 «Si les plantes et les animaux sont privés du langage, c'est parce qu'ils sont emprisonnés chacun dans leur univers environnants, sans être jamais situés dans l'Éclaircie de l'Être. Or, seule cette éclaircie est "monde". Mais s'ils sont suspendus sans monde dans leur univers environnant, ce n'est pas parce que le langage leur est refusé. » (« Lettre sur l'humanisme » dans Questions IV 83).

${ }^{24}$ Sur la querelle avec Rilke, voir aussi « Pourquoi des poètes? », dans Chemins qui ne mènent nulle part 343.

25 «Cette solidarité de l'être (de l'étant) et de l'être de l'homme, peut-on seulement en discuter, aussi longtemps que la pensée demeure attachée au concept de l'homme admis jusqu'ici? » («Qui est le Zarathoustra de Nietzsche? », dans Essais et conférences, 144); «De tout étant qui est, l'être vivant est probablement pour nous le plus difficile à penser, car s'il est, d'une certaine manière, notre plus proche parent, il est en même temps séparé par un abîme de notre essence ek-sistante. En revanche, il pourrait sembler que l'essence du divin nous fût plus proche que cette réalité impénétrable des êtres vivants [...] que la parenté 
corporelle avec l'animal, de nature insondable, à peine imaginable» (« Lettre sur l'humanisme », dans Questions IV 82).

26 « Aucun mot du langage n'est ce qu'il est en raison d'un contexte purement physique, en raison d'un processus naturel - comme c'est le cas, par exemple, chez l'animal où un cri est déclenché en raison d'un état physiologique quelconque. [...] Les sons inarticulés que les animaux émettent indiquent certes quelque chose; les animaux peuvent même - comme on a coutume de dire, bien que ce ne soit pas juste - se comprendre entre eux. Mais aucune de ces

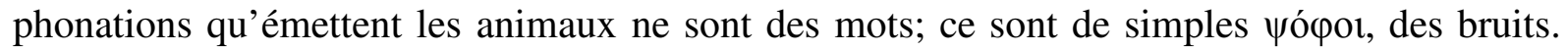
Ce sont des phonations vocales ( $\varphi \omega v \eta ́)$ auxquelles manque quelque chose, à savoir la signification. Par son cri, l'animal ne désigne pas et ne comprend pas » (GA 29/30, 443).

\section{Ouvrages cités}

Agamben, G., L'ouvert. De l'homme et de l'animal, tr. J. Gayraud, Paris, Payot et Rivages, 2002.

Aristote, De l'âme, tr. J. Tricot. Paris, Vrin, 1972.

Burgat, F., Liberté et inquiétude de la vie animale, Paris, Kimé, 2006.

Caron, M., Heidegger. Pensée de l'être et origine de la subjectivité, Paris, Cerf, 2005.

Ciocan, C., «Sur le concept de pulsion (Trieb) chez Heidegger », dans Essays in Celebration of the Founding of the Organization of Phenomenological Organizations, publication en ligne : www.o-p-o.net, 2003.

Dastur, F., « Pour une zoologie "privative” ou comment ne pas parler de l'animal? », Alter, $\mathrm{n}^{\circ} 3$ («L'animal »), 1995, 281-317.

—, Heidegger et la question anthropologique, Louvain-Paris, Peeters, 2003.

Derrida, J., De l'esprit. Heidegger et la question, Paris, Galilée, 1987.

—, «L'animal que donc je suis », dans L'animal autobiographique, Paris, Galilée, 1999

—, « Il faut bien manger ou le calcul du sujet », dans Points de suspension, Paris, Galilée, 1992.

Fontenay, E. de, Le silence des bêtes. La philosophie à l'épreuve de l'animalité, Paris, Fayard, 1999.

Franck, D., «L'être et le vivant », Philosophie, n 16, Paris, Minuit, 1987.

Gadamer, H.-G., Les chemins de Heidegger, tr. J. Grondin, Paris, Vrin, 2002. 
Glendinning, S., « Heidegger and the Question of Animality », International Journal of Philosophical Studies, $\mathrm{n}^{\circ} 4$ (1), 1996, 67-86.

Haar, M., « Dasein et animalité », dans Le Chant de la terre, Paris, L’Herne, 1987.

Heidegger, M. Gesamtausgabe (GA), Vittorio Klostermann Verlag, Frankfurt am Main.

—, Platon : Le Sophiste [GA 19; 1924/1925], tr. J.-F. Courtine et al., Paris, Gallimard, 2001.

—, Prolégomènes à l'histoire du concept de temps [GA 20; 1925], tr. A. Boutot, Paris, Gallimard, 2006.

—, Conférences de Cassel [1925], tr. J.-C. Gens, éd. bilingue, Paris, Vrin, 2003.

-, Concepts fondamentaux de la philosophie antique [GA 22; 1926], tr. A. Boutot, Paris, Gallimard, 2003.

—, Etre et temps [GA 1; 1927], tr. E. Martineau, Paris, Authentica, 1985.

—, Les problèmes fondamentaux de la phénoménologie [GA 24; 1927], tr. J.-F. Courtine, Gallimard, 1985.

—, The Metaphysical Foundations of Logic [GA 26; 1928], tr. M. Heim, Bloomington, Indiana U. P., 1984.

—, Les concepts fondamentaux de la métaphysique. Monde, finitude, solitude [GA 29/30], tr. D. Panis, Paris, Gallimard, 1992.

—, Aristote, Métaphysique $\Theta$ 1-3 [GA 33; 1931], tr. B. Stevens et P. Vandevelde, Paris, Gallimard, 1991.

—, De l'essence de la vérité [GA 34; 1931-1932], tr. A. Boutot, Paris, Gallimard, 2001.

—, Qu'est-ce qu'une chose? [GA 41; 1935-1936], tr. J. Reboul et J. Taminiaux, Paris, Gallimard, 1988.

—, Schelling [GA 42; 1936], tr. J.-F. Courtine, Paris, Gallimard, 1993.

—, Question I et II, Paris, Gallimard, «Tel », 1990.

—, Questions III et IV, Paris, Gallimard, «Tel », 1990.

—, Parmenides [GA 54; 1942-1943], tr. A. Schuwer et R. Rojcewicz, Bloomington, Indiana U. P., 1992. 
—, Chemins qui ne mènent nulle part, Paris, Gallimard, « Tel », 2001.

—, Qu'appelle-t-on penser? [GA 8; 1951-1952], tr. A. Becker et G. Granel, Paris, PUF, « Quadrige », 1999.

—, Essais et conférences [GA 7], tr. A. Préau, Paris, Gallimard, «Tel », 1997.

Hegel, G. W. F., La raison dans l'histoire (1837), tr. K. Papaioannou, UGE, «10/18 », 1965.

Houillon, V., « Pauvrement habite l'animal... », Alter, n 3 (« L'animal »), 1995, 115-150.

Kacem, M. B, L'affect, Paris, Tristram, 2004.

Krell, D.F., Daimon Life. Heidegger and Life-Philosophy, Indianapolis, Indiana U. P., 1992.

McNeil, W., « Life Beyond the Organism. Animal Being in Heidegger's Freiburg Lectures », dans P. H. Steeves (dir.), Animal Others. On Ethics, Ontology and Animal Life, SUNY Press, 1999, 197-248.

Richir, M., «L'animalité selon Heidegger », dans Phénomènes, Temps et Êtres. Ontologie et phénoménologie, t. 2, Grenoble, Jérôme Millon, 1988.

Safranski, R., Heidegger et son temps, Paris, Grasset, 1996.

Scheler, M., La situation de l'homme dans le monde, tr. M. Dupuy, Paris, Aubier Montaigne, 1951.

Sommer, C., Heidegger, Aristote, Luther. Les sources aristotéliciennes et néo-testamentaires $d$ 'Être et temps, Paris, PUF, «Épiméthée », 2005.

Revue germanique internationale, «Trieb : tendance, instinct, pulsion », n 18, PUF, 2002.

White, C. J., Time and Death. Heidegger's Analysis of Finitude, Burlington, Ashgate, 2005. 